care, and doctors (especially when self-employed) have to convince people of their competence and care (Steinkopf 2012; 2017). Further, the rituals and structure of contemporary medicine still play an important role in highlighting and legitimizing patients' need for care. Although health belief systems have largely changed from spiritual to biological, parallels between contemporary medicine and shamanism are evident. Although medical training no longer requires celibacy, the arduous and prolonged training can be considered a similarly transformative event. Interestingly, the duration of medical training in Western medicine has remained at six or seven years since medieval times at least, despite great differences in availability of useful biomedical knowledge (French 2003). Further, patients usually go to doctors with the expectation of receiving acknowledgment of their sick states by being prescribed treatments, often leading to the prescription of pure or impure placebos or even overtreatment (e.g., Faessler et al. 2010; Howick et al. 2013; Meissner et al. 2012). Although the prescribed treatment is ineffective or even harmful, it contributes to the legitimization of the patient's suffering as well as to the doctor's reputation. On the one hand, the doctor's acknowledgement of the patient's sick state manifested in diagnosis and prescription is a signal to the community for a legitimate need for relief and care. On the other hand, a doctor generously prescribing treatment and thus acknowledging the patient's needs may develop a better reputation (and more economic success) than a medical miser who treats only when treatment is indicated (Fabrega 1997; Steinkopf 2017).

In sum, the shaman has to persuade patients and onlookers through a healing spectacle, and the sick individual has to convince others of a sick state, incapacity, and a need for care. Both may have a shared interest in making the healing encounter appear effective, because it supports the shaman's claim of expertise and the patient's adoption of the sick role. Modern doctors also act and compete in terms of satisfying expectations and building community consensus around the sick person's need for care.

\section{Toward a neurophysiological foundation for altered states of consciousness}

\section{doi:10.1017/S0140525X17002187, e87}

\section{Shadab Tabatabaeian and Carolyn Dicey Jennings University of California, Merced, CA 95343 \\ stabatabaeian@ucmerced.edu_cjennings3@ucmerced.edu http://faculty.ucmerced.edu/cjennings $3 /$}

\begin{abstract}
Singh's cultural evolutionary theory posits that methods of inducing shamanic altered states of consciousness differ, resulting in profoundly different cognitive states. We argue that, despite different methods of induction, altered states of consciousness share neurophysiological features and cause shared cognitive and behavioral effects. This common foundation enables further cross-cultural comparison of shamanic activities that is currently left out of Singh's theory.
\end{abstract}

Singh's cultural evolutionary theory successfully accounts for multiple aspects of shamanism as a recurrent phenomenon. When addressing the evolution of shamanism, however, Singh rejects altered states of consciousness (ASC) as a crucial element, considering these less important than other elements, such as behavioral adaptations. He further claims that different methods of inducing ASC have "profoundly different physiological and psychological effects" (sect. 4.2, para. 1). One of Singh's reasons for setting aside ASC as explanatory is that he thinks the ASC used by shamans do not have a common neurophysiological basis. Yet, this claim is unsubstantiated. Here, we suggest that ASC both share certain neurophysiological features and give rise to shared cognitive and behavioral effects.
Altered states of consciousness induced by methods as varied as sensory deprivation, shamanic drumming, trance, meditation, endurance running, hallucinogen consumption, and even epileptic seizures produce shared cognitive and behavioral effects, including hallucinations, out-of-body experiences, ego dissolution, enhanced imagery, and a distorted sense of time (see Castillo 1990; Danielson et al. 2011; Dietrich 2003; Forgays \& Forgays 1992; Hayashi et al. 1992; Kjellgren et al. 2003; 2008; Mason \& Brady 2009; Speth et al. 2016; Suedfeld 1980; Suedfeld \& Eich 1995; Vaitl et al. 2005; Zuckerman \& Cohen 1964). These shared effects are not likely to be coincidental; rather, it is likely that ASC share certain neurophysiological features that correspond to these cognitive and behavioral effects. Although there is as of yet no consensus as to what these shared neurophysiological features are, it is worth reviewing some evidence supporting the view.

One line of evidence comes from using electroencephalography (EEG) to compare ASC with non-altered states. EEG results indicate that ASC, regardless of induction method, correspond to greater activity in the low-frequency bands-delta, theta, and slow alpha (see Takahashi et al. [2005], Batty et al. [2006], Cahn \& Polich [2006], and Fox et al. [2013] for meditation and relaxation states; Neher [1962], Oohashi et al. [2002], and Gingras et al. [2014] for trance and shamanic drumming; Hayashi et al. [1992] and Iwata et al. [2001] for sensory deprivation; Muthukumaraswamy et al. [2013], Tagliazucchi et al. [2016], and Carhart-Harris et al. [2016] for hallucinogens; and Danielson et al. [2011] for epileptic seizures). These frequency bands, in turn, are associated with internally directed attention and attenuated interaction with the external environment (e.g., Benedek et al. 2014). These results suggest that internally directed attention might be a common element of ASC. Supporting this view, practices such as drumming, trance, endurance running, and focused meditation simulate sensory deprivation to varying degrees and cause highly focused internal attention, such that engagement with external stimuli is highly attenuated (Castillo 1990; Dahl et al. 2015; Dietrich 2003; Gingras et al. 2014; Hove et al. 2016; Lutz et al. 2008). This disengagement of attention from external stimuli, known as perceptual decoupling, helps to sustain internally directed tasks (Hove et al. 2016; Smallwood et al. 2007; Smallwood et al. 2011; Spreng et al. 2010). Similarly, hallucinogens that induce ASC impair reactions to external stimuli, but this occurs because of the failure of sensory gating in filtering out extraneous stimuli, causing a failure to process incoming information adequately (Carter et al. 2005; Geyer \& Vollenweider 2008).

The second line of evidence comes from studies using neuroimaging techniques such as functional magnetic resonance imaging (fMRI). Such studies suggest that one of the most common effects of ASC, known as ego dissolution (the loss of a sense of self), corresponds to disruptions to the default mode network $(\mathrm{DMN})$. The DMN is activated during self-referential processes, such as autobiographical memory retrieval (Boly et al. 2008; Buckner et al. 2008; Raichle et al. 2001). Studies using fMRI have indicated that, during ASC induced by various methods including focused meditation (Brewer et al. 2011), hallucinogen consumption (Carhart-Harris et al. 2012; 2016; Palhano-Fontes et al. 2015; Tagliazucchi et al. 2016; Speth et al. 2016), and epileptic seizures (Danielson et al. 2011) - the activation of the DMN decreases, giving rise to ego dissolution. These studies also reveal that, during ASC, regardless of induction method, high functional connectivity is exhibited between three interacting brain networks: default mode, frontoparietal control, and salience (see Brewer et al. 2011; Carhart-Harris et al. 2016; Hasenkamp et al. 2012; Hove et al. 2016; Tagliazucchi et al. 2016). The frontoparietal control network is responsible for cognitive control and attention (Cole \& Schneider 2007; Cole et al. 2014; Vincent et al. 2008). The salience network is responsible for detecting salient events (internal or external) and for directing resources to the relevant neural areas (Christoff et al. 2009; Menon \& Uddin 2010; Seeley et al. 2007). Enhanced connectivity among these three 
large-scale brain networks could explain some of the effects experienced by individuals during ASC, including the perceptual decoupling discussed above.

This evidence supports the idea of a common neurophysiological foundation to ASC. We further claim that a common neurophysiological foundation to ASC can be of significant explanatory value for a cultural evolutionary theory of shamanism. For example, Singh's theory notes but does not currently explain the shaman's insensitivity to pain, which, in our view, would be explained by perceptual decoupling in ASC. Similarly, Singh's theory does not account for the shaman's ritualistic practice of using dark times of the day or dark spaces, such as caves; in our view, this is explained by the relationship between sensory deprivation and ASC. Finally, Singh's theory notes but does not currently explain why shamans believe they are not in control of their experience, which, in our view, is explained by the experience of ego dissolution common to ASC. Although ASC and their underlying mechanisms require further investigation, we argue that it is too soon to reject the idea of a common neurophysiological foundation to ASC. Our argument is consistent with the primary objectives of Singh's cultural evolutionary theory and can be considered an extension to that theory, because it can help explain the universality, ubiquity, and endurance of shamanic activities around the world.

\section{The social functions of shamanism}

doi:10.1017/S0140525X17002199, e88

\section{Rachel E. Watson-Jones and Cristine H. Legare Department of Psychology, The University of Texas at Austin, Austin, TX 78712. \\ dr.rachel.watsonjones@gmail.com legare@austin.utexas.edu www.rachelwatsonjones.com www.cristinelegare.com}

\begin{abstract}
Cultural evolutionary accounts of shamanism must explain the cross-cultural recurrence and variation in associated practices. We suggest that Singh's account of shamanism would be strengthened by considering the social functions of shamanism in groups. Shamanism increases social group cohesion, making it distinct from other magicoreligious practices.
\end{abstract}

The term shamanism can be applied broadly to a wide set of magico-religious practices that occur across cultures. Historical, social, economic, and ecological factors contribute to how shamanistic practices are organized within cultural groups. Singh's account of the cultural evolution of shamanism provides a framework for explaining cross-cultural variation and recurrence of related practices. As the author notes, however, the current "theory is agnostic as to whether shamans provide benefits to clients or groups" (sect. 3.4, para. 3). We argue that understanding social functions is central to a cultural evolutionary account of shamanism. We discuss how the social nature of shamanism differentiates it from practices related to other magico-religious practices such as witchcraft. We also explain that the rituals associated with shamanism provide a means of social cohesion that does not always necessarily involve signaling cooperative intent (WatsonJones \& Legare 2016).

Shamanism and traditional healing are distinct from practices related to witchcraft. Winkelman's (1986b) analysis of Standard Cross-Cultural Sample (Murdock \& White 1969) data provided evidence that magico-religious practices are related to political and economic complexity. This analysis revealed that there are four major practitioner types related to socioeconomic conditions:

1. The Healer Complex that includes shamans, shaman/ healers, and healers - the major distinction among these being that the shamans and shaman/healers engage in trance states, whereas the healers do not. Shamans were most associated with hunter/gatherer societies, shaman/healers with all societies that had agriculture, and healers with almost all societies that were politically integrated.

2. Mediums who were characterized as engaging in possessionlike trance states and were found in agricultural and pastoralist societies

3. Priests who were often leaders in societies with political integration and a hierarchical social structure that relied heavily on agriculture

4. Malevolent practitioners who engaged in harmful activities against others in societies with political integration. Across many cultures, witchcraft practitioners are considered malevolent actors. For example, in South Africa, witchcraft is a common attribution for the ultimate cause of AIDS transmission (Legare \& Gelman 2008). Witchcraft practitioners are accused of using their supernatural knowledge for individual gain and are not regarded as working in the interests of all members of the community. In many cases, once communities with shamans are integrated into more complex socioeconomic systems, practitioners associated with the predominant religion call out or label shamans as witches to delegitimize their role within the community (Winkelman 1986b).

Shamanism is communal. Many anthropologists have argued that shamanism has social functions; it increases social cohesion and cooperation. Shamans act as a conduit to the supernatural realm, where they act on group members' behalf and enable rituals (often dramatic) that bind group members together for a common goal. This is not to say that shamans act selflessly. As the author notes, shamans often are prestigious members of their communities. An account of the cross-cultural recurrence and variation of magico-religious practices must take into account the perceived, or actual, motivations of the practitioners. The shaman reduces uncertainty surrounding important fitnessrelated events, such as illness, childbirth, hunting, and protection from enemies. Healers are almost exclusively responsible for dealing with illness-related issues, and witches are perceived as focused on causing harm to others, most often surreptitiously. It would be interesting to consider whether shamanism is ever practiced in secret - if shamanistic practices are done in isolation from other community members, are they ever done without request or knowledge of the community?

The importance of shamanism and similar practices for group functioning is not necessarily related to signaling cooperative intent, and the author provides a cogent argument against costly signaling accounts of shamanism. Cooperative intent doesn't get off the ground without a preexisting, regularly maintained core of social cohesion. The rituals associated with shamanism provide a means of reaffirming social solidarity through shared experience and providing an indication of commitment to group norms.

Does shamanism professionalize because of its contribution to group functioning? Singh argues that shamanism professionalizes "because individuals typically must invest in transformative practices to be considered capable of influencing uncertain outcomes" (sect. 5.1, para. 4). In this way, the professionalization differs from that of practices related to technical knowledge. Both expertise and group consensus increase perceptions of ritual efficacy (Souza \& Legare 2011). We argue that the professionalization of shamanism also is related to the function it serves for groups. As the earliest ritual practitioners, shamans provided a means of group cohesion that was, and is, essential for small-scale societies.

The defining characteristics of shamanism come partly from its relationship to social group dynamics and the benefits its practices provide for social cohesion. Singh's framework of the cultural evolution of shamanism neglects an essential explanatory feature of shamanism: the social benefits it provides to human groups. 